\title{
Health Assessment using Raspberry Pi
}

\author{
Ann Maria Sunny ${ }^{1}$, Ann Paul ${ }^{2}$, Ann Susan Jose ${ }^{3}$, Cuckoo Anitha Joseph ${ }^{4}$, Anu Rani Philip ${ }^{5}$ \\ Electronics and Communication Engineering Dept., VJCET, Ernakulum, India ${ }^{1,2,3,4,5}$
}

\begin{abstract}
Health assessment using Raspberry Pi is a continuous monitoring system at home for assessing early health changes to address the management of chronic conditions as people age. Sensors embedded in the body and environment capture various parameters of health. Changes in those parameters are detected as potential signs of changing health. If changes are detected, an alert message will be sent. Activities will be investigated using in-home sensors both day and night to ensure security. The sensor data will be then sent to mobile devices through the Internet. Also a continuous monitoring can be done through the webpage.
\end{abstract}

Keywords: sensors, data, monitor, webpage.

\section{INTRODUCTION}

This paper is based on the idea of generating automated health alerts using in-home sensors. The concept has been realized in such a way that it is cost effective. Thus we have developed a health monitoring system using minimal number of sensors.

\section{THEORY}

In this paper, embedded health assessment, on-going assessment of health changes are done on the basis of individual's behavior, activity patterns and baseline health conditions. Sensors embedded in the environment are used to collect behavior and activity patterns for the purpose of detecting health changes.

\section{A. How do these Sensors work?}

Passive infrared (PIR) motion sensors will be used to capture activity in a particular location in the home, e.g., in the patient's room. The pattern of room to room activity will be studied as a means of investigating health changes. Motion density from PIR motion sensors (i.e., number of events per unit time) can capture overall activity level that may be linked to health condition.

In addition, significant effort using in-home sensing has focused on safety, e.g., fall detection. There is also work on wearable sensor networks and sensing incorporated into clothing. Some wearable systems have been studied extensively or advanced to the stage of commercialization, e.g., accelerometers for fall detection.

The PIR motion sensors, which use the wireless X-10 protocol for data transmission, generate an event every seven seconds if there is continuous motion. This is used as an artifact to capture the general activity level in the home by computing a motion density as motion events per unit time. For example, a person with a sedentary lifestyle may generate only 50 motion events per hour, whereas a person with a very active life style may generate 400 or more motion events per hour.
B. Analysis of Sensor data

The logged sensor data are automatically analyzed, looking for changes in an individual's data patterns. If a change is detected, an alert message is sent. The health alert message is displayed in a web portal in addition to the sensor data, which facilitates fast access to the sensor data visualization. This helps to determine whether the alert is relevant for this person from a clinical perspective.

\section{BLOCK DIAGRAM}

Our system consists of 2 parts:

- Wearable unit

- Node devic

\section{A. Wearable Device}

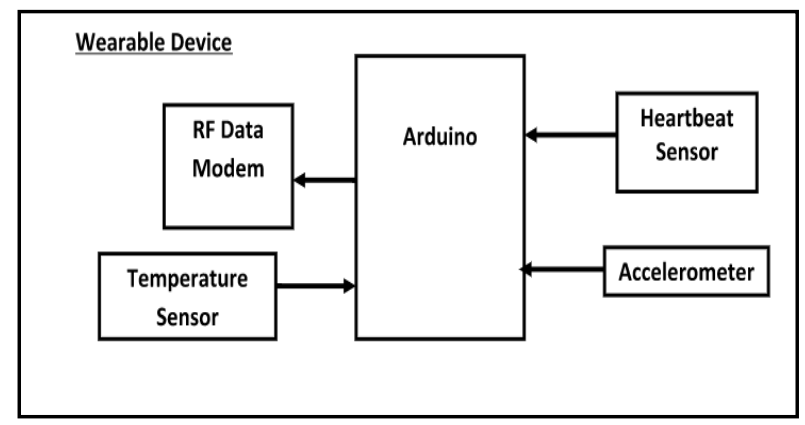

Figure 1. Block diagram of wearable device

Temperature sensor, heartbeat sensor and accelerometer are the sensors that are placed at the patient's body. These sensors sense the patient body parameters like heartbeat, temperature and detect any falls. The sensed data from the sensors are sent to the sensor node and are received at the sensor node. Sensor node is the controller which control signals received from sensors and converts the received analog signal into digital signal. Thus sensor node is nothing but the controller. In this system, Arduino controller is used as the sensor node. Main functions of the sensor node are. 
- Control of signal

- Analog to digital conversion

- Transmission of digital signal

RF data modem is used to send the digital signals wirelessly from Arduino controller to Raspberry Pi

\section{B. NodeDevice}

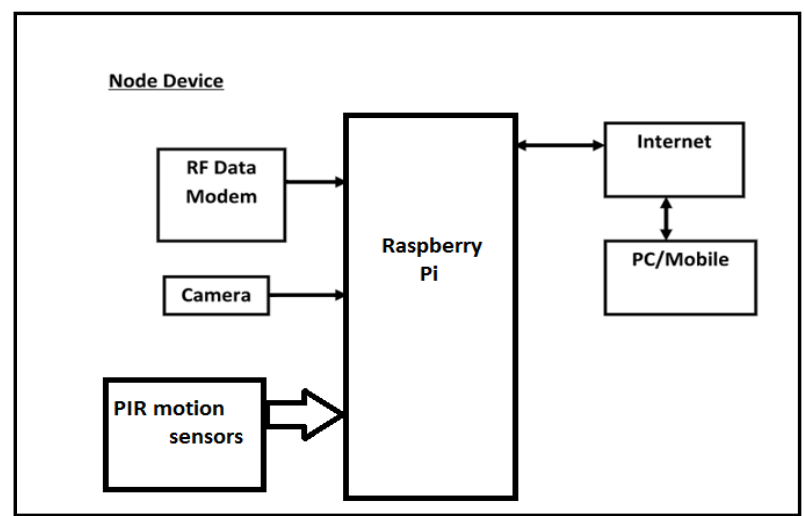

Figure 2. Block diagram of node device

The digital signals from the Arduino are received at the Raspberry Pi using RF data modem. Raspberry Pi cannot process analog signals since it does not have in built ADC. So analog signals from the sensors are converted into digital signal using Arduino controller and then sent to the Raspberry Pi. Raspberry Pi then send the received signals data to mobile devices through the internet. Also the camera attached to the Raspberry Pi is used to monitor the activities in the room through the webpage.

\section{IV.FLOW CHART}

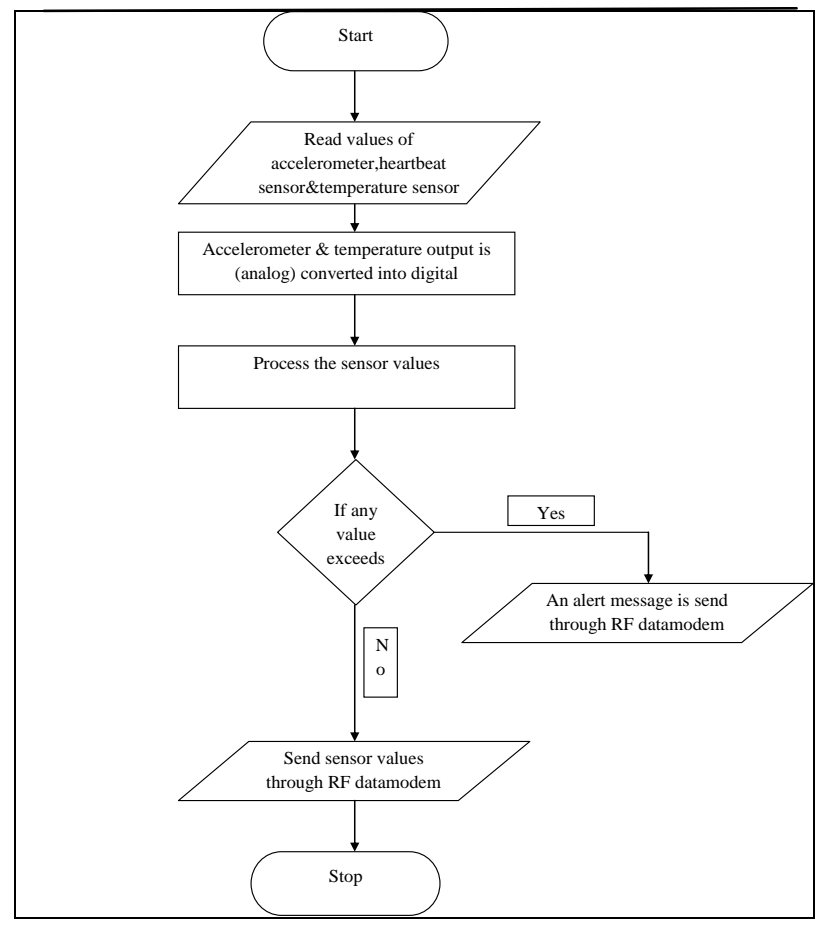

Figure 3. Flowchart of wearable device

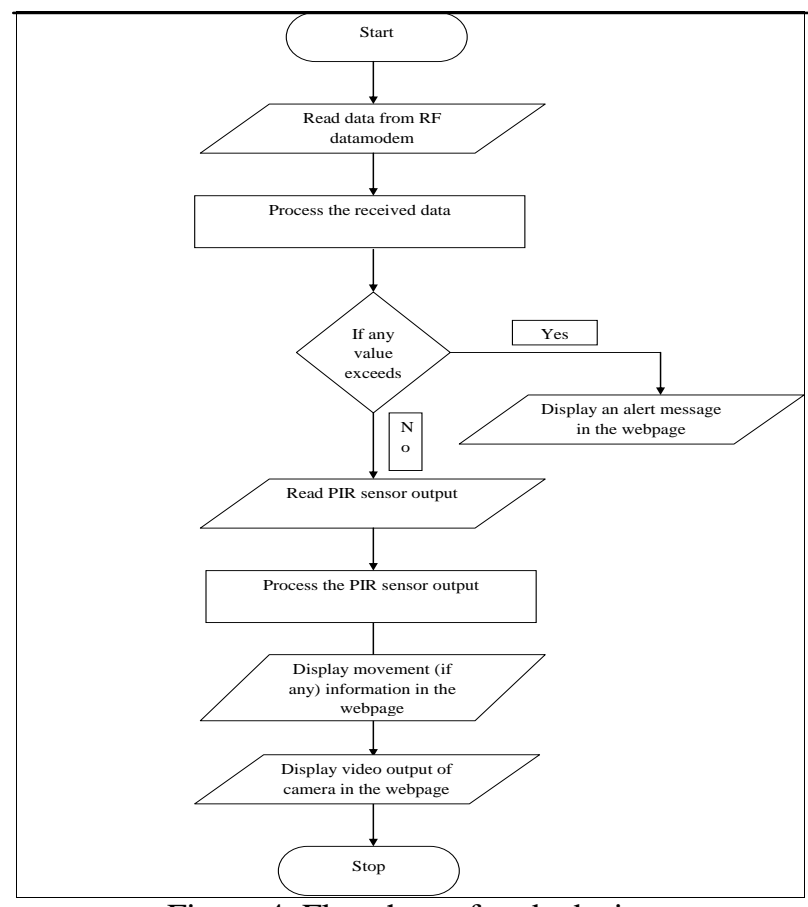

Figure 4. Flowchart of node device

\section{V.CONCLUSION}

The devices were successfully tested. Wearable device collected the output of sensors placed in patient's body, which were processed and sent to the node device. If there were any deviation from normal values, an alert message was also sent using RF datamodem. Received sensor values and alert messages were displayed in the webpage. PIR sensors detected human presence (if any). Visualization of the room was also available from the camera output in the webpage. This presents a system which makes it possible to monitor a person's health from a distant location and hence could be used to assess the health status of elderly people who are alone at home. All the peripherals used in this paper are easily available. So the manufacturing and replacement is much easier. This is also comparatively a cheaper device. All the components, except the temperature sensor, are very accurate in their operation. Temperature sensing could be made more precise using a thermometer by noting the difference between its initial and final readings using a circuit, instead of LM 35, in the future.

\section{REFERENCES}

[1] Automated Health Alerts Using In-Home Sensor Data for Embedded Health Assessment: IEEE Journal of Translational Engineering in Health and Medicine, Date of publication: 10 April 2015.

[2] Monitoring and Detection Platform to Prevent Anomalous Situations in Home Care, Article, Published: 5 June 2014.

[3] A review of wearable sensors and systems with application in rehabilitation, Journal of Neuro Engineering and Rehabilitation, Published: 20 April 2012

[4] Accelerometry: providing an integrated, practical method for long-term, ambulatory monitoring of human movement, Institute of Physics Publishing Published: 16 February 2004.

[5] An Introduction to MEMS, PRIME Faraday Partnership, Wolfson School of Mechanical and Manufacturing Engineering, Lough borough University, Loughborough, Published in 2002. 